\title{
Observation of Virus-Like Particles in Vascular and Coelomic Hemolymph of Riftia pachyptila
}

\author{
R. R. Helton, * D. H. Powell,* S. C. Cary, ** S. W. Polson, *** K. J. Czymmek* \\ * DBI Bio-Imaging Center, Delaware Biotechnology Institute, 15 Innovation Way, Room 117, \\ University of Delaware, Newark, DE 19711, USA \\ **University of Waikato, Gate 1 Knighton Road, Private Bag 3105, Hamilton 3240, New \\ Zealand \\ ***Department of Plant and Soil Sciences, Delaware Biotechnology Institute, 15 Innovation \\ Way, Room 148, University of Delaware, Newark, DE 19711, USA
}

Deep-sea hydrothermal vent ecosystems are amongst the few distinctive environments where microbial chemolithotrophy is the prevailing means of developing macro faunal communities. Among those fauna, the giant tubeworms, Riftia pachyptila, live and thrive in the extreme environment relying on the symbiotic bacteria in their gut to obtain nutrients from the surrounding waters given that sunlight is not an available resource [1-2]. These giant tubeworms are considerably large, often reaching 3 meters in length while dwelling in waters over 2000 meters deep. It is also now generally recognized that viruses are the most copious members of marine microbial communities [3-4], and now are also being recognized as significant participants in these extreme locations [5]. Here, we investigate potential connections between the microbial and macro faunal communities of the hydrothermal vents.

Vascular and coelomic hemolymph (blood analogue) was removed from several Riftia pachyptila (giant tubeworm) collected by the submersible Alvin during a research cruise onboard the $R / V$ Atlantis along the East Pacific Rise in the Pacific Ocean during November 2008 (Fig. 1 A - B). The hemolymph was examined for virus-like particles, in addition to other microbial organisms, using a Zeiss CEM 902 transmission electron microscope.

The hemolymph was first $0.45 \mu \mathrm{m}$ filtered to remove large particles prior to observation. Formvar-carbon support copper grids (Electron Microscopy Sciences, cat\# FCF400-Cu) were treated in two ways: 1) grids were touched to the filtered hemolymph for $1 \mathrm{~min}$, rinsed two times in sterile $\mathrm{dH}_{2} \mathrm{O}$ and stained with $5 \%$ ammonium molybdate for $10 \mathrm{sec}$; 2) grids were placed on filter paper inside polyallomer tubes and centrifuged at 25,000xg for $1 \mathrm{hr}$ with the filtered hemolymph diluted with $1 \%$ phosphate buffered saline. After centrifugation, the grids were stained as previously described. Preliminary transmission electron microscopy data revealed two putative viral-like particles. One particle type measured $58 \mathrm{~nm}$ in width and $84 \mathrm{~nm}$ in length, with distinct defined edges (Fig. 2A). The second particle was larger, with overall dimensions of $147 \mathrm{~nm}$ (Fig. 2B). Our initial finding of these two particles is encouraging and further investigations of the hemolymph to characterize additional virus-like particles are underway.

The preliminary microscopy evidence presented here is the first observation of virus-like particles within the vascular and coelomic hemolymph of the hydrothermal vent giant tubeworms, Riftia pachyptila. This suggests that viruses are in the hemolymph, and supports the current hypothesis that viruses also play a significant role in the extreme geothermal environment of the deep-sea. 
References

[1] C.M. Cavanaugh, et al., Science 213 (1981) 340.

[2] H. Felbeck, Science 213 (1981) 336.

[3] R. W. Hendrix, Curr Opin Microbiol 6 (2003) 506.

[4] K. E. Wommack, et al., Appl Environ Microbiol 58 (1992) 2965.

[5] S. J. Williamson, et al., ISME J. 2 (2008) 1112.
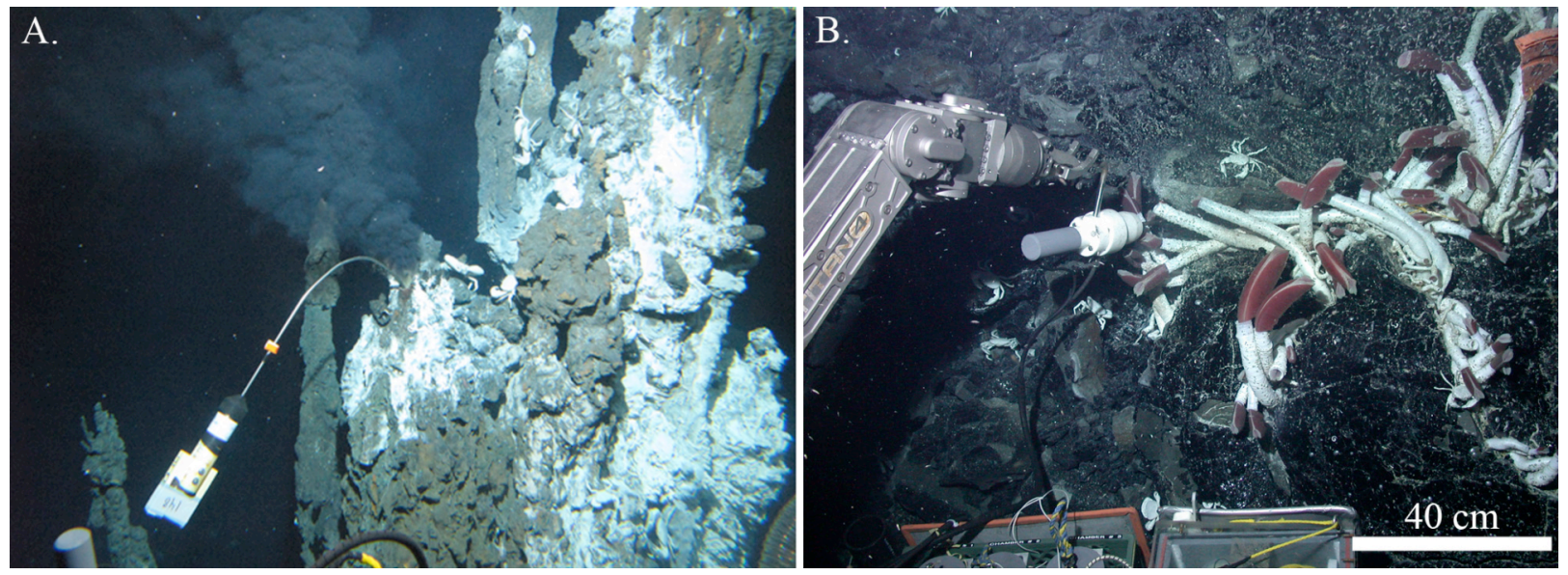

Fig. 1. Deep-sea hydrothermal vent. A. Black smoker vent, upper area where nutrients are released into the water. B. Alvin arm collecting Riftia pachyptila giant tubeworms at the base of the black smoker hydrothermal vent.
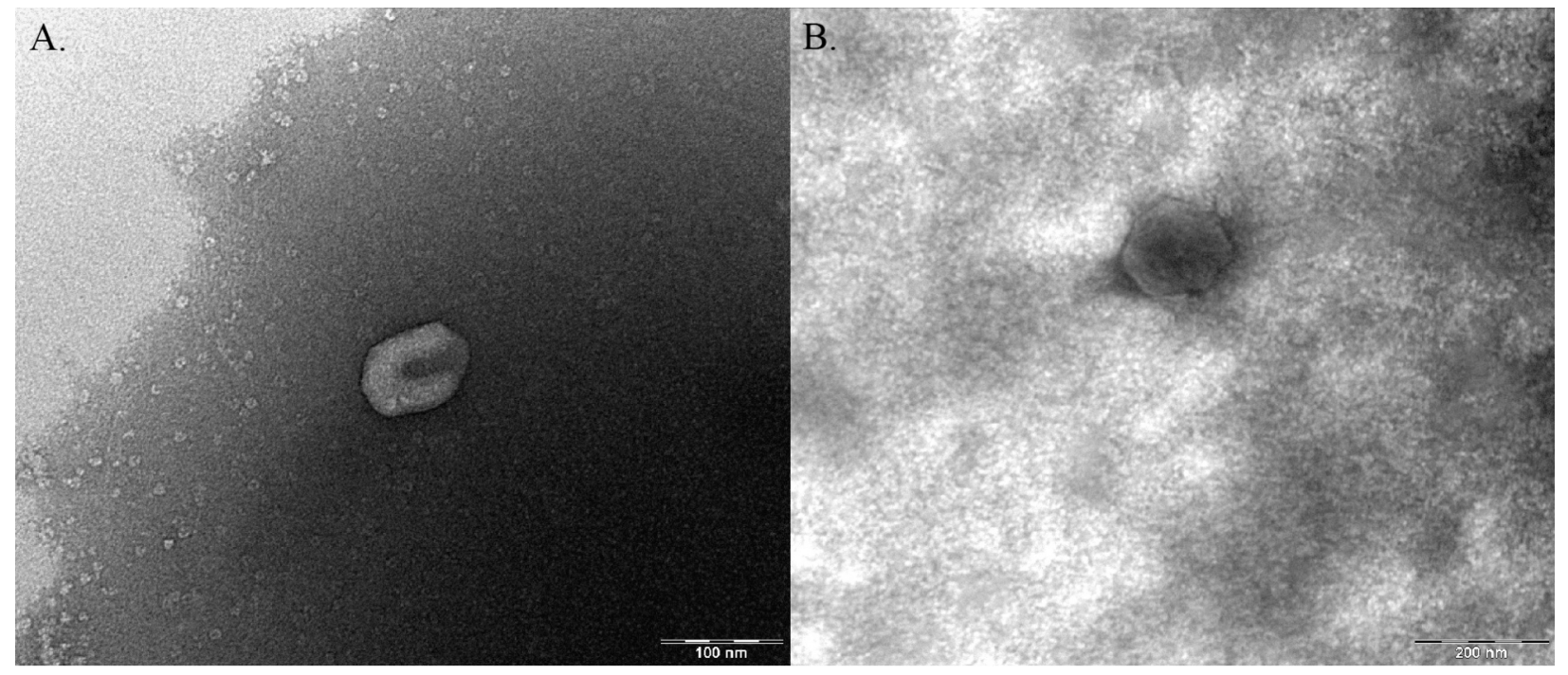

Fig. 2. Virus-like particles observed via transmission electron microscopy. A. Putative virus particle from grid touched to the filtered hemolymph, showing capsid dimensions of $58 \mathrm{~nm} \times 84$ $\mathrm{nm}$, scale bar is $100 \mathrm{~nm}$. B. Putative virus particle from grid centrifuged with filtered hemolymph showing capsid diameter of $147 \mathrm{~nm}$, scale bar is $200 \mathrm{~nm}$. 